\title{
DIRETRIZES SUSTENTÁVEIS NA CONCEPÇÃO DE PRODUTOS
}

\author{
Cecília da Rocha Pessôa \\ Universidade Federal de Pernambuco \\ ceciliar.pessoa@gmail.com \\ Kátia Medeiros de Araújo \\ Universidade Federal de Pernambuco \\ katiaa@terra.com.br \\ Renata Garcia Wanderley \\ Universidade Federal de Pernambuco \\ renatagw@hotmail.com
}

Resumo: A partir de revisões das metodologias para o desenvolvimento de produtos sustentáveis elaboradas por Kazazian (2005), Manzini e Vezzoli (2011), Fletcher e Grose (2011); dos princípios sobre a ressignificação de artefatos de Cardoso (2012); das elaborações de Oliveira (2013), das estratégias de design para a sustentabilidade de Salcedo (2014); são apresentadas, por meio de compilação, os principais direcionamentos para a concepção de artefatos de forma sustentável. Três grandes domínios são propostos: ambiente, economia e sociedade. Para trabalhá-los, também três focos são apontados: natureza, pessoas e continuidade. Cada um desses parâmetros exige algumas ações básicas para alcançá-los. O presente artigo pretende apresentar a construção de um dispositivo de análise (compilação das diretrizes) que servirá para compreensão da perspectiva sustentável dos designers e do papel desses profissionais nas empresas de vestuário (dissertação do autor).

Palavras-chave: design, diretrizes, sustentabilidade, metodologias de ecodesign.
Abstract: From reviews of the methodologies for the development of sustainable products developed by Kazazian (2005), Manzini and Vezzoli (2011), Fletcher and Grose (2011); the principles on reframing Cardoso artifacts (2012); elaborations of Oliveira (2013), the design strategies for sustainability of Salcedo (2014); are presented by means of compilation, the main directions for the conception of artifacts in a sustainable way. Three main areas are proposed: environment, economy and society. To work them, also three focus are identified: nature, people and continuity. Each of these parameters requires some basic actions to achieve them. This paper intends to present the construction of an analysis device (compilation of guidelines) that will serve to the understanding the perspective sustainable 
of designers and the role of these professionals in the apparel companies (Author's dissertation).

Keywords: design, guidelines, sustainability, eco-design methodologies.

\section{INTRODUÇÃO}

Repensar o papel do designer continua sendo uma atitude necessária, mesmo após 45 anos das primeiras discussões sobre as atividades deste profissional em relação ao meio ambiente e a qualidade de vida das pessoas. Neste sentido, ainda é um grande desafio para os designers projetar funcionalidades e significados a um objeto, para que, por exemplo, o descarte não seja o seu destino final.

A lógica do sistema de produção e consumo de artefatos da sociedade contemporânea vem saturando os recursos da natureza e causando desastres ambientais graves. Muitas empresas estão desenvolvendo produtos ecologicamente corretos, porém os produtos 'verdes' continuam inseridos no mesmo paradigma mercadológico: o aumento da produção de artefatos, para estimular o consumo e obter mais lucro. Esse modelo necessita ser revisto porque os recursos naturais não são infinitos. No setor do vestuário, muitas empresas possuem discursos sustentáveis. Porém muitas práticas referendadas de ecodesign não resolvem o complexo conjunto de questões relativas ao meio ambiente. Essas práticas muitas vezes elegem somente um dos fatores para praticar. Como por exemplo, a escolha de matérias primas renováveis, que por si só, não resolvem o problema ambiental; simplesmente amenizam os danos à natureza.

Dessa forma, na sustentabilidade, o conceito difundido sobre desenvolvimento sustentável consiste em satisfazer as demandas atuais dos indivíduos (necessidades e desejos) sem comprometer a qualidade de vida das gerações futuras. E atua em três dimensões: econômica, social e ambiental (SACHS, 2009; KAZAZIAN, 2005). Para Sachs (2009), o crescimento econômico tem que favorecer o meio ambiente e os seres humanos e não continuar destruindo o capital natural. Portanto, se o padrão produtivo da sociedade continuar sendo praticado sem a lógica da sustentabilidade, as condições básicas para o futuro dos cidadãos serão comprometidas.

Desde o ano de 1971, o designer e educador Victor Papanek iniciou a defesa do design social e ecologicamente correto. A reflexão de Papanek (1984) estava orientada para as questões sociais. Este pensamento era fundamentado no verdadeiro papel do design direcionado para atender as reais necessidades dos indivíduos, principalmente as minorias: deficientes, pobres, excluídos por meio da inovação e da criatividade. 0 autor defende projetos sem patentes, pois é contrário à obtenção de lucro a partir das necessidades dos indivíduos. Neste sentido, o autor reflete a questão da sustentabilidade centrada na ecologia, na cultura e nas necessidades humanas, descartando assim, o fator econômico. De acordo com Sachs (2009), as posições extremas entre o economicismo arrogante e o fundamentalismo ecológico foram descartadas. E concorda com a abordagem de ecodesenvolvimento fundamentada na harmonização de objetivos sociais, ambientais e econômicos, se estiver direcionada através de oito critérios: social, cultural, ecológico, ambiental, territorial, econômico, da política nacional e política internacional. Para o autor, é necessário ter como objetivo alcançar os direitos plenos de cidadania para toda a população, como também para as gerações futuras e, principalmente, reconceitualizar o desenvolvimento. E 
conceitua o desenvolvimento "[...] como apropriação efetiva de todos os direitos humanos, políticos, sociais, econômicos e culturais, incluindo-se aí o direito coletivo ao meio ambiente" (SACHS, 2009, p. 60).

Atualmente, diversos autores (como MANZINI-2008 e 2015; FLETCHER e GROSE-2011; CARDOSO-2012; VEZZOLI-2010; MANZINI e VEZZOLI-2011) continuam defendendo a importância de se repensar o papel do profissional de design para a sustentabilidade. Para tanto, construíram teorias, metodologias, entre outros conhecimentos e dispositivos visando a orientação da produção e consumo sustentável.

O designer italiano Manzini (2008) defende que para melhorar a qualidade de vida dos indivíduos, as próprias pessoas terão que modificar hábitos no estilo de vida e consumir menos. Para o autor a transição rumo à sustentabilidade será um processo de aprendizado social. Com base neste pensamento, o profissional de design deve ser considerado como uma ferramenta estratégica para executar novas práticas de concepção e produção para estimular novas relações mais sustentáveis com os objetos.

Manzini e Vezzoli (2011) identificam alguns dos novos comportamentos sociais das empresas para conscientização acerca dos problemas ambientais na sociedade: o tratamento de poluição, a utilização de tecnologias limpas (redução do ato de poluir nos sistemas produtivos) e o redesenho dos produtos e serviços. Os autores conceituam ecodesign como projeto orientado por critérios ecológicos. Dessa forma, 0 desenvolvimento de produtos limpos requer não somente o uso de tecnologias limpas, mas de uma nova capacidade de design. Neste sentido, o design pode influenciar novas mudanças no comportamento do usuário sem utilizar sofisticadas tecnologias e com soluções aceitáveis. Entretanto, para os autores, melhorar o que já existe não é suficiente para atingir a sustentabilidade. É necessário pensar em produtos e serviços diferentes dos que existem hoje (MANZINI; VEZZOLI, 2011).

Neste cenário, esse estudo aponta como problema de pesquisa o processo não sustentável da produção de artefatos de moda. Com isso, questiona: (a) Como construir produtos de moda de forma sustentável? (b) Quais os principais parâmetros a serem trabalhados para a produção sustentável? (c) Que ações e estratégias podem tornar a produção de moda mais sustentável? (d) As normatizações de sustentabilidade já apresentadas são suficientes e aplicáveis?

Diante dessa problematização, o objetivo principal deste estudo é a construção de diretrizes bases de sustentabilidade para a concepção de produtos de moda. Para tanto, buscou: (a) compilar as normatizações (as leis, normas e planos) oficiais na área de sustentabilidade, (b) mapear os parâmetros de sustentabilidade apontados na área de design e (c) os principais domínios de atuação da sustentabilidade.

Para tanto, desenvolveu pesquisa teórico-reflexiva, construindo conhecimentos acadêmicos (as diretrizes base da sustentabilidade) e se estruturando em teorias sobre sustentabilidade. Qualifica-se como pesquisa aplicada, uma vez que os conhecimentos desenvolvidos visam sua utilização real. Portanto, estruturou-se principalmente em pesquisa bibliográfica. 


\section{ALGUNS ESTUDOS SOBRE SUSTENTABILIDADE}

Diversos pesquisadores de diferentes áreas vem trabalhando a sustentabilidade enquanto meta (fim), processo (meio) e/ou princípio (início) de ação. Mesmo com focos múltiplos, cada estudo já aponta parâmetros importantes para a sustentabilidade, orientando a construção de suas diretrizes básicas.

\subsection{Kazazian (2005)}

Para Kazazian (2005), a natureza é fonte de inspiração para a sociedade. Assim sendo, seus principais eixos de pensamento sobre sustentabilidade foram guiados por meio da natureza: a) interdependência, os processos e as relações dependem uns dos outros; (b) tempo, como uma forma de alongar a duração de vida dos produtos, pois a durabilidade descreve o tempo da relação entre o homem e o objeto" (KAZAZIAN, 2005, p. 44); (c) ciclo, como fluxo fechado, controlando a totalidade do ciclo de vida do produto, em que "a matéria nunca é destruída, mas transformada" (KAZAZIAN, 2005, p. 50); (d) 'optimum', consideração da melhor otimização possível em todas as relações, sendo movimentos que estão sempre em harmonia.

\subsection{Thackara (2008)}

As estratégias de Thackara denominadas por ele de 'design consciente' para concretizar futuros sustentáveis se refletem em: (a) pensar nas consequências das ações antes de executá-las, levando em consideração o contexto das ações de design nos sistemas naturais, industriais e culturais; (b) pensar nos fluxos de materiais e energia de todos os sistemas de projetos; (c) priorizar o ser humano; (d) entregar valor às pessoas e não entregar pessoas aos sistemas; (e) tratar o conteúdo como algo que se faz, não que se vende; (f) tratar a diferença cultural, local e de tempo como fatores positivos; e (g) concentrar em serviços, não em coisas, evitando produzir artefatos sem sentido.

\subsection{Manzini e Vezzoli (2011)}

Manzini e Vezzoli desenvolveram pesquisas e trabalhos no Politécnico de Milão sobre estratégias para projetar e desenvolver produtos sustentáveis. Para esses autores, os requisitos ambientais para o desenvolvimento de produtos e serviços são: (a) minimização dos recursos, redução dos consumos de matéria e energia em todas as fases (atividades de projeto e gestão); (b) escolhas de recursos e processos de baixo impacto ambiental; (c) otimização da vida dos produtos, para que perdurem; (d) extensão da vida dos materiais, valorizando a reaplicação dos materiais descartados; (e) facilidade de desmontagem, facilitando a separação das partes e dos materiais de um artefato.

\subsection{Fletcher e Grose (2011)}

Fletcher e Grose (2011) apontam nove oportunidades de inovação que buscam uma visão mais ecológica de mundo. Estas são ideias que almejam a reflexão dos designers sobre o aprimoramento das suas práticas atuais. As estratégias são: (a) adaptabilidade: criação de peças para serem transformadas pelos usuários para intensificação do uso (como roupas com múltiplas funções, atemporais e modulares); (b) vida útil otimizada: a durabilidade de uma vestimenta em termos materiais, estilísticos e emocionais (c) usos de baixo impacto (como na lavagem e secagem de peças); (d) serviços e compartilhamento (como no reparo, aluguel, design open source); (e) local, "passar para uma escala menor de atividade muda as relações entre 
materiais pessoas, lugares, comunidades e meio ambiente" (FLETCHER; GROSE, 2011, p. 106); (f) biomimética: imitar (entender e aplicar) os padrões e estratégias da natureza como guia para o design de produtos; (g) velocidade: a flexibilidade para adaptação de diferentes contextos; (h) necessidades, peças de indumentária que possam satisfazer as necessidades (abrigo e proteção) e desejos (expressão pessoal e pertencimento) dos consumidores; (i) engajamento, reflete o conceito de conexidade das coisas, por exemplo o design colaborativo, com o engajamento tanto do designer quanto do usuário.

\subsection{Cardoso (2012)}

Cardoso (2012) recomenda alguns princípios para serem considerados no processo de significação dos artefatos: (a) reversibilidade, o design para o desmonte com a utilização de módulos, para diminuir o acúmulo de lixo e estender a sobrevida através de substituições e combinações de partes; (b) manutenção, o artefato inserido em um sistema de uso, para aperfeiçoamento, reaproveitamento e simplificação dos processos de substituição; (c) pensamento sistêmico, conhecimento do todo e suas relações com as partes do sistema (integração ao contexto); (d) durabilidade, mais ênfase ao sentido do objeto do que aos materiais, objetos considerados heranças e relíquias.

\subsection{McDonough e Braungart (2013)}

O arquiteto William McDonough e o químico Michael Braungart são os criadores da abordagem 'Cradle to Cradle' (2013). O pensamento deste modelo consiste em ver o descarte como alimento, como nutriente. O desperdício não deve existir nesse sistema. Os criadores aplicam métodos de cooperação entre indústria e consumidores, para assim, modificar a forma tradicional de pensar os resíduos. Um conceito defendido pelos autores é o upcycled (valorizar o ciclo) em que o nutriente biológico é isolado, respeitando sua qualidade original (ciclos biológicos e tecnológicos). McDonough e Braungart (2013) indicam cinco princípios orientadores para os designers e os líderes empresariais: (a) sinalizar sempre a intenção para o novo paradigma, (b) restaurar, (c) estar pronto para inovar mais, (d) entender e se preparar para o aprendizado e (e) exercer a responsabilidade Inter geracional. Estes princípios tem o foco nas pessoas.

\subsection{Oliveira (2013)}

A pesquisa de Oliveira (2013) apresenta maneiras de como o design poderá contribuir para solução de problemas socioeconômicos e ambientais. Dessa forma, o autor criou a metodologia de design sistêmico denominada 'F.L.O.R.A.'. Esse método foi criado para ser aplicado no 'Pólo de Confecções de Pernambuco', porém, pode ser utilizado como referência por designers em qualquer âmbito corporativo ou territorial. As cinco guias de referência do método F.L.O.R.A. são: (a) ' $F$ ' de feedback, ciclos de produção fechados; (b) ' $L$ ' de ligação, cooperação entre os diversos atores: designers, empresas e stakeholders; (c) ' $\mathbf{O}$ ' de otimização, eficiência nos processos de produção (manufatura enxuta e princípios da biomimética); (d) ' $R$ ' de renovação, estratégia de diferenciação na comunicação institucional; (e) ' $A$ ' de adaptação, a flexibilização corporativa (nichos de mercado e/ou oferta de serviços). 


\subsection{Salcedo (2014)}

Salcedo assinala oito estratégias de design para a sustentabilidade: (a) pensar na desmontagem da peça: design para a reciclagem; (b) pensar em criar laços emocionais (ex: sistemas de traçabilidade); (c) pensar no bem-estar social de todos os atores envolvidos no desenvolvimento das peças; (d) pensar em minimizar o desperdício: design sem resíduos; (e) pensar na durabilidade da peça para retardar a necessidade de conserto e facilitar o processo de reparo; (f) pensar no papel do usuário nas fases de uso e manutenção (recomendações em etiquetas); (g) pensar em aumentar a vida útil do produto (peças modificáveis, multifuncionais ou reversíveis e oferecer a opção de aluguel de peças); e, por último, (h) pensar na gestão de resíduos, tanto nas etapas de produção como para o fim da sua utilização.

\section{A COMPILAÇÃO DAS DIRETRIZES}

No levantamento dos estudos dos respectivos autores citados anteriormente sobre a temática da concepção de artefatos utilizando requisitos, métodos e estratégias de sustentabilidade, foi observado três direcionamentos macro nas diversas diretrizes projetuais relativos à regeneração da natureza, às intervenções dos seres humanos (co-participação) e à durabilidade dos artefatos. Essas orientações visam a busca para um estilo de vida mais sustentável, não mais orientado para o aumento desenfreado da produção e do consumo de artefatos.

Estes direcionamentos indicam os três contextos principais da sustentabilidade. Eles explicitam as motivações e organizam os principais parâmetros apontados por cada autor.

Diante dessa contextualização, essa pesquisa define três parâmetros macro de atuação para a sustentabilidade: (a) natureza (ambiental), (b) pessoas (social), (c) continuidade (econômico). Com isso, define o processo de projetar de forma sustentável exigindo também 03 ações: (1) 'Projetar integrando a natureza'; (2) 'Projetar com as pessoas'; e (3) 'Projetar para continuidade'.

O resultado, a nosso ver, contribui para que o conjunto de diretrizes seja assimilado de forma mais sistemática, responsável e inteligente pelos profissionais de design, enfatizando-se a importância do todo (conceitos macros) e sua relação com as partes (orientações específicas), e assim, facilitando-se o surgimento de novas percepções dos profissionais no ato de projetar.

O quadro 1 esquematiza todo o processo de compilação das diretrizes. Ele relaciona os três contextos, as motivações e os parâmetros dos autores, relacionandoos com grupos de ação das diretrizes compiladas: 
Quadro 1 - Compilação das diretrizes: dimensões da realidade abordados

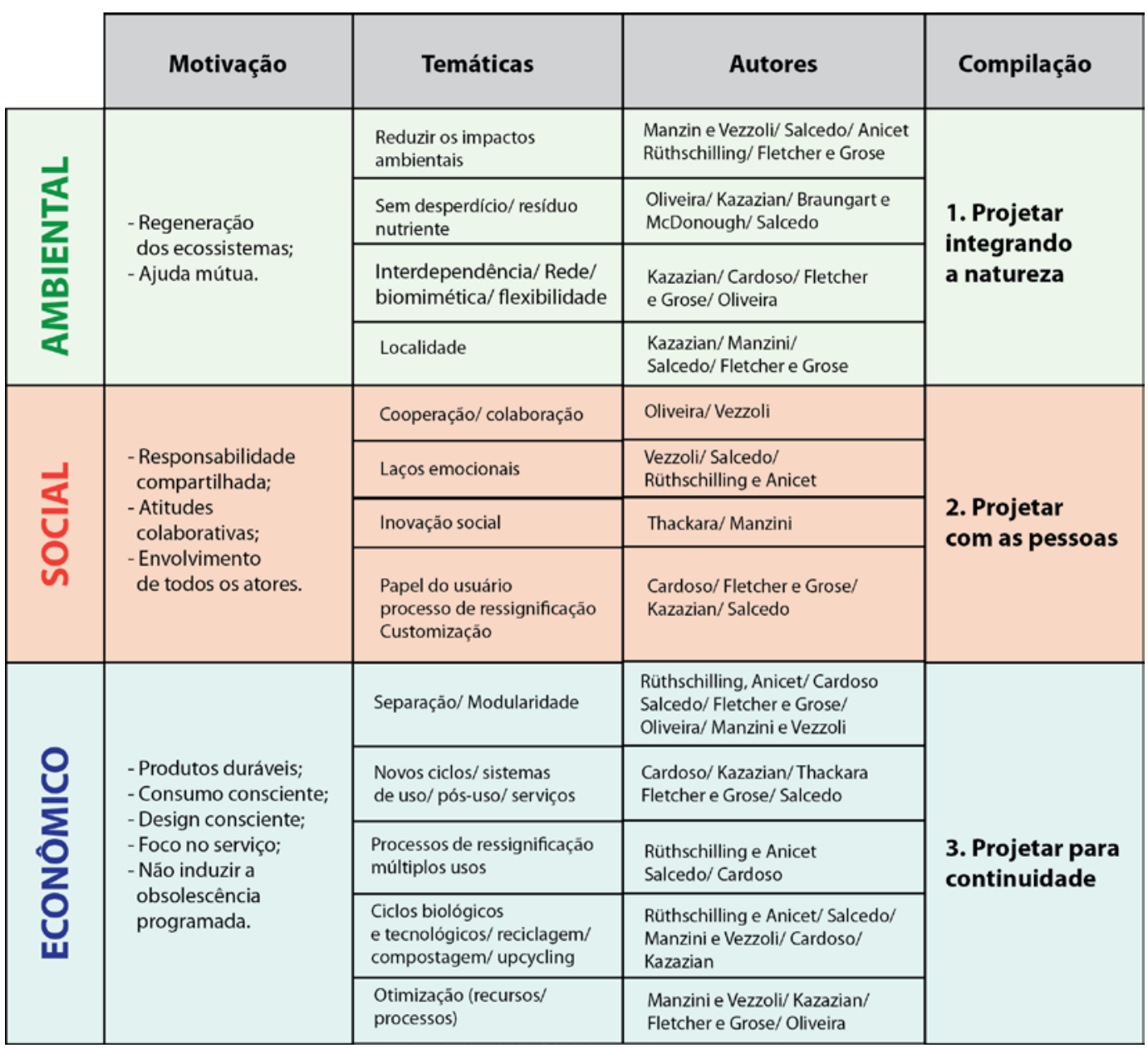

Fonte: "Elaborado pelo autor, com base na pesquisa realizada".

\subsection{Projetar integrando a natureza}

'Projetar integrando a natureza' é o primeiro princípio norteador para o desenvolvimento responsável de produtos e serviços. Sua ideia base é associar o desenvolvimento com a prosperidade mútua da natureza e também das pessoas, as quais fazem parte dela, respeitando-se a resiliência dos ecossistemas para a evolução da vida no planeta Terra. Os processos ecológicos da natureza são cíclicos e flexíveis, então são sistemas fechados e que se adaptam ao contexto. Portanto, o objetivo é se pensar em estratégias que respeitem os limites do meio ambiente na fase inicial do projeto.

No direcionamento de 'projetar integrando a natureza', os principais fatores inseridos neste pensamento projetual são: (a) reduzir os impactos ambientais na escolha de recursos e processos, (b) projetar sem desperdício, e (c) trabalhar a localidade, explorando o contexto local. Ao valorizar as potencialidades das matérias primas, dos contextos (natureza/ cultura/ comunidade) poderá surgir novas formas de regenerar os recursos ambientais e sociais de um determinado local.

Quando se promove a redução das perturbações ambientais na escolha do uso dos recursos, respeita a resiliência dos ecossistemas, o tempo certo para a natureza se regenerar (extração/ crescimento/ cultivo). Utilizar de maneira mais adequada 
(reduzindo, reutilizando e reciclando) matérias primas e energia envolvidas em todo processo de ciclo de vida de um produto ou serviço (entradas e saídas) é a estratégia de ação. Como exemplo são assinalados: minimizar a quantidade de material na fabricação de uma peça de vestuário, a energia necessária e as embalagens; otimizar os consumos do transporte; e minimizar o consumo de recursos durante o sistema de uso, como com o uso coletivo, eficiência no funcionamento e na manutenção.

Nos sistemas da natureza não tem desperdício, todos os resíduos são reaproveitados na alimentação de outros ciclos biológicos. Para reproduzir esse contexto, as sobras e refugos devem ser considerados nutrientes de ciclos fechados biológicos e tecnológicos para regenerar os sistemas produtivos. Projetar evitando as perdas no processo de produção é ação base.

Projetar pensando no local contribui para mudança nas relações entre materiais, pessoas, lugares e meio ambiente. Este pensamento fortalece a identidade e o pertencimento de indivíduos. Por exemplo, a utilização de recursos disponíveis em um determinado local (como fibras tradicionais, regionais, orgânicas) estimula a cadeia produtiva local. Esta estratégia colabora à princípio para a redução do consumo de energia devido à redução da logística dos transportes, valoriza a mão de obra local (habilidades dos moradores e artesãos locais) e respeita os ecossistemas naturais do local.

\title{
3.2. Projetar com as pessoas
}

O segundo direcionamento é projetar com as pessoas. Ele significa pensar em estratégias para valorizar todos os atores envolvidos em todas as etapas do ciclo de vida de um produto. Para Thackara (2008), é necessário utilizar mais sensibilidade no pensar, no agir e nas relações. $O$ autor afirma:

\begin{abstract}
Precisamos promover novas relações fora das nossas zonas de conforto, aprender novas formas de colaborar e conduzir projetos, melhorar a capacidade de todos os cidadãos de se envolver em um diálogo significativo sobre seu ambiente e contexto e promover novos relacionamentos entre as pessoas que fazem as coisas e as pessoas que as utilizam. (THACKARA, 2008, p. 39)
\end{abstract}

No ato projetivo, o designer terá que ser sensível e criativo na inserção de formas para (a) criar envolvimento emocional dos usuários; (b) desenvolver ações colaborativas para construção de parceiros; (c) gerar autonomia para que usuários solucionem seus próprios problemas. Segundo Manzini (2015), trata-se de uma cultura na qual todos projetam. Entretanto, para o autor, os especialistas em design deveriam ser os promotores dessas iniciativas.

A construção de laços emocionais dos usuários nos sistemas de uso e manutenção de produtos permite que os indivíduos se sintam valorizados no processo. Seus atos influenciam nos resultados dos sistemas.

Utilizar, por exemplo, técnicas de cocriação com usuários, para que cada um possa ter a liberdade de adaptação em sua própria vestimenta (modificação ou customização); e/ou sistemas de traçabilidade (rastreamento/ ações colaborativas envolvendo todos os atores envolvidos nas diversas etapas de ciclo de vida dos produtos) fortalecem os vínculos entre a empresa, fornecedores e usuários; 
As empresas também são compostas por pessoas. Portanto, transformar empresas e stakeholders em parceiros, integra-os ao processo. Essa tática torna-os responsáveis também pelos efeitos provocados pela produção e consumo dos produtos.

O pensamento projetual com o foco no ser humano corrobora o argumento de Manzini (2008) sobre a transição rumo à sustentabilidade em que as mudanças serão mais comportamentais do que tecnológicas. Se o ser humano não tiver a consciência ambiental enraizada de forma natural em seu cotidiano, em sua filosofia de vida, será muito difícil modificar os padrões de estilo de vida insustentável da sociedade contemporânea.

\subsection{Projetar para continuidade}

O terceiro e último direcionamento refere-se ao 'projetar para continuidade', ampliando a visão de ciclo de vida. Busca desestimular a obsolescência programada, com produtos com duração menor do que a tecnologia permite. A questão não é apenas aumentar a durabilidade no sentido dos materiais, mas principalmente na criação de relações afetivas entre o consumidor e o produto, fazendo com que o objeto possa viver anos com o seu usuário. Para Kazazian (2005) guardamos os objetos em função das relações (utilitárias, hedonistas ou cognitivas) que estabelecemos com eles. Novas ideias podem ser inventadas a partir de necessidades, de desejos e do mercado.

Como estratégias são apontadas: construção de artefatos com sistema de ressignificação constante (produto-usuário); ou como peças legitimadas como obra de arte, valorizando, assim, todos os fatores, estilísticos, materiais e emocionais que influenciam na vida útil de um vestuário.

A continuidade pode se estabelecer por meio de: (a) pensar em novos ciclos de vida, (b) intensificar o uso e (c) adaptação e renovabilidade.

As possibilidades do usuário de criar novos significados com objetos, permite que novos ciclos de vida possam ser produzidos. Criar produtos com múltiplos usos possibilita diversos ciclos de vida.

Projetar para o compartilhamento de peças, uso coletivo, gera a intensificação do uso de um artefatos. A substituição da oferta do produto pela oferta do serviço é outra forma de aumentar o uso de um mesmo produto.

Projetar para adaptação e renovabilidade significa planejar a separação de materiais e partes de um artefato, viabilizar serviços de reparos e manutenção e sistemas de coleta e devolução de peças aos produtores. Propondo atualizações para retardar a obsolescência dos artefatos. Os processos de reciclagem (ciclo fechado de produção), e de upcycling (valorização de materiais descartados) fazem com.

Portanto, ampliar a vida útil de objetos é o grande sentido de projetar para continuidade. E o papel do usuário neste direcionamento é muito relevante. No sentido de adaptação às demandas dos usuários, capacidade de renovação, tanto material quanto simbólica (ressignificação dos artefatos); e co-participação do usuário no processo de forma mais efetiva e consciente. 
A figura 1 esquematiza esse processo de ação enfatizando a importância do todo (conceitos macros) e sua relação com as partes (orientações especificas). Aponta a ação principal "projetar" e seus princípios bases "integrando com a natureza; com as pessoas e para a continuidade". A figura denominada 'criando com o infinito' foi idealizada com base no pensamento para o infinito, a favor das gerações futuras, em que a criatividade também é infinita. O conceito de infinito no processo de sustentabilidade pode ser considerado utópico, mas nesta pesquisa, o sentido de infinito é de não ter limites para criar soluções mais sustentáveis, respeitando sempre os limites de natureza, para que ela possa ser infinita.

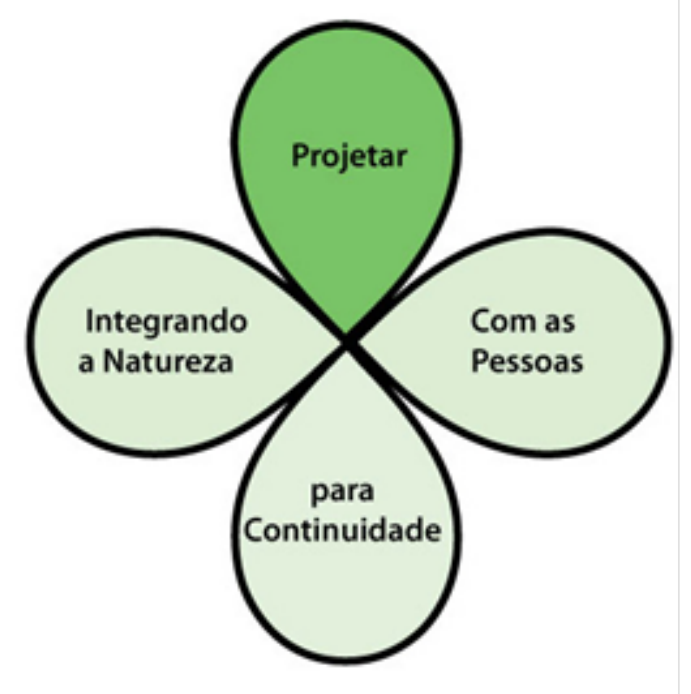

Figura 1: Criando com o infinito: imagem ilustrativa para representar a síntese das diretrizes. "Elaborado pelo autor, com base na pesquisa realizada".

O resultado desta compilação e síntese constituirá o dispositivo de análise dos discursos nas inferências das entrevistas. Como esta é uma pesquisa em design, auxílios visuais como tabelas e imagens facilitam o processo de comunicação de uma mensagem, tanto na rapidez do processo de assimilação quanto na retenção da informação. Portanto, para representar visualmente e conceitualmente esta síntese de diretrizes, foi idealizado como um pensamento para o infinito, a favor das gerações futuras, em que a criatividade também é infinita. O conceito de infinito no processo de sustentabilidade pode ser considerado utópico, mas nesta pesquisa, o sentido de infinito é de não ter limites para criar soluções mais sustentáveis, respeitando sempre os limites de natureza, para que ela possa ser infinita.

Essa forma simples de perceber a sustentabilidade, poderá incentivar o surgimento de novas modos de fazer logo no início da fase projetual. Desse modo, esta consciência poderá ser uma contribuição relevante e educativa para os criadores de vestuário. Esta percepção ampliada de pensar a sustentabilidade propiciará inspiração para um debate infinito sobre as diversas possibilidades de caminhos rumo à sustentabilidade no segmento de design. Para projetar futuros em direção ao infinito.

Para representar o conceito da síntese foi elaborado um gráfico para demonstrar visualmente a mensagem conceitual do infinito nas orientações sustentáveis. 


\section{CONCLUSÕES}

A percepção da necessidade de se trabalhar com a sustentabilidade do designer está clara e consciente. Diversos estudos já indicam ações sustentáveis e orientadores de análise. No entanto, na prática, pouco se encontra de inovações sustentáveis.

$\mathrm{Na}$ verdade, no momento de planejamento dos artefatos os designers tem as ferramentas necessárias para promover mudanças nos comportamentos, nas relações e nas formas de pensar de empresas e consumidores, mas parece não as utilizar. Assim, as pessoas envolvidas nos sistemas de uso de artefatos ou serviços são as executoras das mudanças. Entretanto, poderia ser os designers os principais responsáveis por propiciar tais mudanças.

Observou-se que se os atores envolvidos no processo de produção do produto não tiverem a sensibilidade para entender a essência da sustentabilidade no seu próprio cotidiano, as soluções comprometidas com a natureza, com as pessoas e com a continuidade não serão incorporadas naturalmente. A filosofia e o pensamento do designer devem tentar vencer as regras do mercado (lucro $x$ produção) e das imposições (organizacionais) e limitações (tecnológicas $x$ físicas) das empresas. Assim sendo, as diretrizes construídas por essa pesquisa busca proporcionar para os designers (a) obter um pensamento macro da sustentabilidade (natureza = ambiental/ pessoas $=$ social $/$ continuidade $=$ econômico) e (b) incluir, no ato de projetar, as possíveis relações que o usuário (responsabilidade compartilhada) poderá criar para melhorar sua forma de lidar com os artefatos, para que eles não sejam desprovidos de sentido e sejam destinados ao lixo.

Além dessa aplicabilidade projetual, as diretrizes podem também ser parâmetros de análise para a discussão da prática real da sustentabilidade nos processo de criação (ideia), produção e consumo de produtos. Elas indicam o que deve ser observado antes, durante e depois da produção de artefatos.

\section{REFERÊNCIAS}

CARDOSO, Rafael. Design para um mundo complexo/ Rafael Cardoso. São Paulo: Cosac Naify, 2012.

FLETCHER, Kate; GROSE, Lynda. Moda \& Sustentabilidade: Design para Mudança. Tradução Janaína Marcoantonio. São Paulo, Editora Senac São Paulo, 2011.

KAZAZIAN, Thierry. Haverá a idade das coisas leves: design e desenvolvimento sustentável/ organizado por Thierry Kazazian; traduçãoo de Eric Roland Rene Heneault. - São Paulo: Editora Senac São Paulo, 2005.

MANZINI, Ezio. Design para inovação social e sustentabilidade - Comunidades criativas, organizações colaborativas e novas redes projetuais/ Ezio Manzini; [coordenação de tradução Carla Cipolla; equipe Elisa Spampinato, Aline Lys Silva]. Rio de Janeiro: EPapers, 2008.

MCDONOUGH, William; BRAUNGART, Michael. Cradle to cradle: criar e reciclar ilimitadamente/ William Mcdonough, Michael Braungart; [tradução Frederico Bonaldo].São Paulo: Editora G. Gili, 2013.

OLIVEIRA, Emilio Augusto Gomes de. Design sistêmico e ecoinovação - estratégias sustentáveis aplicadas para o Polo de Confecções de Pernambuco / Emilio Augusto 
Gomes de Oliveira, 2013. 390 f. il. Tese (doutorado) - Universidade Estadual Paulista. Faculdade de Arquitetura, Artes e Comunicação, Bauru, 2013.

PAPANEK, Victor, Design for the real world Human Ecology and Social Change. Thames and Hudson Ltda, 181 A High Holborn, London WC1V 7QX, c1984.

SALCEDO, Elena. Moda ética para um futuro sustentável. [tradução Denis Fracalossi.] Barcelona: Editora G. Gili, 2014

SACHS, Ignacy. Caminhos para o Desenvolvimento Sustentável/ Organização: Paula Yone Stroh. - Rio de Janeiro: Garamond, 2009.

THACKARA, John; Plano B: o design e as alternativas viáveis em um mundo complexo/ John Thackara; tradução Cristina Yamagami. - São Paulo: Saraiva: Versar, 2008.

VEZZOLI, Carlo. Design de sistemas para a sustentabilidade: teoria, métodos e ferramentas para o design sustentável de "sistemas de satisfação"/ Carlo Vezzoli. Salvador: EDUFBA, 2010. 